ORIGINAL

\title{
Reingreso en prisión de reclusos drogodependientes excarcelados a una comunidad terapéutica, en función de variables previas al tratamiento
}

\section{Reincarceration of drug-dependent prisoners released into a therapeutic community, according to pretreatment variables}

\author{
VICTORIA RAMOS BARBERO*; \\ Rodrigo J. CARCEdo GonZÁlez ${ }^{* *}$
}

${ }^{*}$ Universidad de Burgos
${ }^{* *}$ Universidad de Salamanca

Enviar correspondencia a

Victoria Ramos Barbero.

Facultad de Humanidades y Educación. Universidad de Burgos. C/ Villadiego s/n. 09001 Burgos.

Telf. 0034947 258074. Fax 0034901706977

E-mail: vramos@ubu.es

\section{Resumen}

El principal objetivo de este artículo es identificar las variables penales y penitenciarias previas al tratamiento asociadas al reingreso en prisión, en una población de reclusos drogodependientes que fueron excarcelados desde diferentes centros penitenciarios de Castilla y León (España) para sustituir su pena privativa de libertad por el tratamiento en una comunidad terapéutica. La población objeto de estudio fue de 120 internos varones. Las fuentes documentales consultadas para la recogida de información fueron: el protocolo de clasificación y tratamiento, el expediente penitenciario y el expediente social. Estos registros oficiales fueron consultados en el Centro Penitenciario de Burgos, responsable de la custodia de los documentos. Aplicando un análisis estadístico descriptivo mediante tablas de contingencia, las variables previas al tratamiento que mostraron mayor asociación con el reingreso en prisión fueron los antecedentes familiares penitenciarios, la condena total superior a cinco años, los ingresos previos a la condena base, la regresión de grado de cumplimiento, la revocación de la libertad condicional, el quebrantamiento de condena, la finalización de la condena en prisión y, muy especialmente, la edad del primer ingreso entre 16 y 21 años. Se confirma mediante un análisis de regresión logística que la variable edad en que los sujetos ingresaron en prisión por primera vez es de vital importancia por el impacto que la prisión puede tener en edades tempranas. Estos resultados ofrecen un perfil del recluso drogodependiente que mayor riesgo tiene de hacer un mal uso de la medida alternativa a la prisión.

Palabras clave: reingreso en prisión, reincidencia delictiva, reclusos drogodependientes, comunidad terapéutica, medidas alternativas a la prisión.

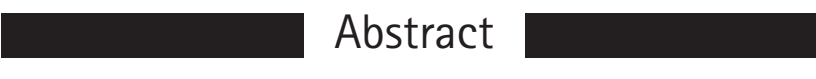

The main aim of this paper is to identify the criminal and penitentiary pretreatment variables associated with re-entry into prison in a population of drug-dependent inmates who had been released from different prisons in Castilla and León (Spain) to finish their prison sentence in a Therapeutic Community. The study population was 120 male inmates. The documentary sources consulted for collecting information were the prison record, the classification and treatment protocol and the social record. These official records were consulted in the Burgos Penitentiary, responsible for custody of the documents. Applying a descriptive statistical analysis with contingency tables, the pre-treatment variables that showed the strongest association with re-entry into prison were family prison history, total sentence exceeding five years, imprisonments prior to the sentence in question, change to a higher security level, revocation of parole, breach of the terms of a sentence, completion of sentence in prison and, especially, age at first entry into prison between 16 and 21 years. It was confirmed through logistic regression analysis that the variable age at which participants entered prison for the first time is critical, given the impact that prison can have at an early age. These results provide a profile of the drug-dependent inmate at greatest risk of making poor use of measures alternative to prison.

Key words: reincarceration, criminal recidivism, drug-dependent prisoners, therapeutic community, alternatives to prison. 
E ste trabajo se centra en el estudio de variables penales, penitenciarias y de conducta objetiva en prisión (e.g., antecedentes familiares penitenciarios, número de ingresos en prisión, condena total formal, quebrantamiento de condena, etc.) de los reclusos que se han mostrado más relacionadas con la reincidencia en el delito. Del mismo modo, este estudio se centra en la reincidencia en un grupo más específico dentro de los internos penitenciarios como son los reclusos drogodependientes excarcelados a una comunidad terapéutica (C. T.). Esta reincidencia en el delito después de su excarcelación al centro de tratamiento ha supuesto su reingreso en prisión.

El actual Reglamento Penitenciario (Real Decreto 190/1996) desarrolla en su artículo 182 el cumplimiento de la pena en un centro extrapenitenciario de deshabituación de la drogodependencia como medida alternativa a la privación de libertad en fase de ejecución penitenciaria, para personas reclusas y drogodependientes. A partir de julio de 2003 la aplicación de esta medida se ha visto afectada por la exigencia del cumplimiento del periodo de seguridad y el pago de la responsabilidad civil (Ministerio del Interior, 2004). No obstante en la actualidad la flexibilización de la aplicación de estas exigencias ha generado un aumento de los internos que se encuentran en disposición de acceder al tratamiento en una $C$. T., haciendo más necesario aún nuestro trabajo, así como otros que evalúen factores asociados a la reincidencia de reclusos drogodependientes (Leganés, 2005; López, 2006).

El debate entre una política criminal más orientada al uso de la prisión y otra más favorable a las penas alternativas continúa abierto (Cid, 2007; González, 2009, Tournier, 2007). Por ello parece especialmente relevante evaluar la efectividad de las excarcelaciones en tercer grado a un centro extrapenitenciario de tratamiento de la drogodependencia, de cara a identificar aquellos factores que más se relacionan con la reincidencia. De tal manera que cuando la Junta de Tratamiento de un determinado centro penitenciario tenga que elaborar un juicio sobre un caso concreto podrá basarse en explicaciones científicas y en variables con consistencia.

La investigación criminológica general sobre predictores de riesgo ha identificado dos tipos diferentes de factores de riesgo de reincidencia delictiva (Andrews y Bonta, 2003; Garrido, Stangeland y Redondo, 2001; Gendreau, Little y Goggin, 1996). En primer lugar, los factores estáticos, o factores de riesgo que son aspectos del pasado del delincuente que no pueden ser modificados por el tratamiento (e.g., edad del primer ingreso en prisión, delincuencia en la familia, etc.). En segundo término, los denominados factores dinámicos 0 factores del sujeto o de su entorno que aún pueden ser modificados (e.g., hábitos laborables, tiempo de permanencia en C. T., creencias favorables a la delincuencia, etc.) y que por tanto sirven como objetivos adecuados para el tratamiento.

A nivel internacional contamos con investigaciones que concluyen que comprometerse con el tratamiento en $\mathrm{C}$. T., es una de las variables más importantes que se asocia con tasas más bajas de consumo de drogas y de reingreso en prisión, así como mayor empleabilidad (Bahr, Masters y Taylor, 2012; Fiestas y Ponce, 2012; Sacks, Chaple, Sacks, McKendrick y
Cleland, 2012; Sacks, Sacks, McKendrick, Banks y Stommel, 2004; Sullivan, McKendrick, Sacks y Banks, 2007). Todos estos estudios aportan evidencia de los beneficios del tratamiento residencial en $\mathrm{C}$. T. postprisión.

Investigaciones realizadas en prisiones estadounidenses analizan la influencia del historial delictivo sobre el reingreso en prisión de reclusos en tratamiento en C. T. En concreto Wexler, Falkin y Lipton (1990) concluyen que la edad de excarcelación y la gravedad del expediente delictivo (medida por el número de ingresos en prisión, años de encarcelamiento, tipo delictivo, sentencias en libertad vigilada y revocación de la libertad condicional) tienen una importante relación con ser de nuevo arrestado. La relación entre ser de nuevo detenido y el expediente delictivo anterior puede deberse a que los delincuentes más peligrosos son arrestados antes que otros. Y la relación entre protagonizar un nuevo arresto y la edad de excarcelación indica que los delincuentes más mayores tardan más en reincidir. Estos resultados son confirmados por investigaciones posteriores (Burdon, Messina y Prendergast, 2004; Inciardi, Martin y Butzin, 2004; Wexler, De Leon, Thomas, Kressel y Peters, 1999; Wexler, Melnick, Lowe y Peters, 1999).

Por otro lado hay dos instrumentos ampliamente utilizados con delincuentes adultos que pretenden predecir el riesgo de reincidencia, son el Psychopathy CheckList Revised (PCL-R, Escala de Evaluación de la Psicopatía) de Robert Hare y el Level of Service Inventory Revised (LSI-R, el Inventario de Nivel de Servicio Revisado) de Andrews y Bonta, que integran ítems de factores estáticos y dinámicos (Stangeland y Redondo, 2001). Por ello consideramos importante seguir investigando sobre estos factores de riesgo.

La revisión bibliográfica del tema nos aporta muy pocos trabajos en el estado español, que analicen la reincidencia posterior al tratamiento, de muestras de reclusos drogodependientes y excarcelados a una C. T. extrapenitenciaria. Hasta donde nuestro conocimiento alcanza, tan sólo existen en nuestro país dos estudios que investigaban factores estáticos (Roca y Caixal, 2002, 2005) y uno sobre factores dinámicos (Ramos y Carcedo, 2010). Debido a esta escasez, también hemos tenido en cuenta otros trabajos que han estudiado estos factores con población general penitenciaria, que no necesariamente son reclusos drogodependientes y no han tenido tratamiento extrapenitenciario en C. T. (Luque, Ferrer y Capdevila, 2004a, 2004b, 2005; Pérez y Redondo, 1991 Redondo, Funes y Luque, 1994; Serrano, Romero y Noguera, 2001).

Con respecto a los factores dinámicos, Ramos y Carcedo (2010) estudiaron una serie de variables que habian sido evaluadas en otros estudios con reclusos y que habian mostrado su relación con la reincidencia como son la evolución terapéutica del sujeto, la motivación para el cambio, la percepción y valoración por parte del paciente del tratamiento (Elzo, Huete, Laespada y Santibáñez, 1995; Fernández, 2005; Fernández y Rico, 2004; Gañán y Gordon, 1999; Menéndez y Rico, 2004). Ramos y Carcedo (2010) encontraron que la ausencia de hábitos laborales, la patología infecciosa por $\mathrm{VIH}$, los niveles altos de extraversión, la finalización del tratamiento extrapenitenciario en $\mathrm{C}$. T. con expulsión o abando- 
no y el menor tiempo de permanencia en la C. T. se asociaron significativamente con una mayor reincidencia.

En referencia a los factores estáticos, la reincidencia ha sido asociada con la presencia de antecedentes familiares penitenciarios (Becedóniz, Rodríguez, Herrero, Paíno y Chan, 2005; Bravo, Sierra y Del Valle, 2009; Serrano, Romero y Noguera, 2001), una condena total formal más larga (Luque, Ferrer y Capdevila, 2004a, 2004b; Redondo et al., 1994; Roca y Caixal, 2002, 2005), un mayor número de ingresos en prisión previos (Luque et al., 2004; Redondo et al., 1994; Serrano, Romero y Noguera, 2001), una edad más joven en el primer ingreso en prisión (Garrido et al., 2001; Luque et al., 2004a, 2004b; Paíno, Rodríguez y Cuevas, 1996; Redondo et al., 1994; Roca y Caixal, 2002, 2005; Serrano, Romero y Noguera, 2001), la existencia de alguna regresión de grado de cumplimiento, revocación de la libertad condicional o bien algún quebrantamiento de condena (Redondo et al., 1994; Roca y Caixal, 2002, 2005; Serrano et al., 2001) y la finalización de la condena por extinción de condena (Luque et al., 2004a, 2004b, 2005). La finalidad de este estudio es, por tanto, investigar estas variables estáticas y comprobar si se asocian o no de manera significativa con la reincidencia.

\section{Método}

\section{Participantes}

La población objeto de estudio está formada por todos los sujetos que fueron derivados en tercer grado a C. T. extrapenitenciaria a lo largo del periodo 1985 - 2003 bajo la responsabilidad del Centro Penitenciario de Burgos. Estos internos provenían de diferentes centros penitenciarios de Castilla y León. La población final obtenida fue de 120 participantes varones, no se llevó a cabo ningún proceso de selección, se trabajó con todos los sujetos que fueron derivados a C. T. Esta población se obtuvo de la revisión de expedientes penitenciarios y protocolos de clasificación de tratamiento de un volumen total de 3654 sujetos que en algún periodo temporal desde 1985 a 2003 habían cumplido condena bajo la responsabilidad del Centro Penitenciario. Estos documentos se encontraban archivados en el Centro Penitenciario de Burgos y en ellos constaba la información sobre concesión y evolución de la medida alternativa a la pena privativa de libertad. Por tanto de los 3654 expedientes revisados encontramos que a 120 sujetos, el Centro Directivo de Instituciones Penitenciarias les concedió esta medida alternativa.

La edad de primer ingreso en prisión oscilaba entre los 16 y 41 años $(M=21.71, D T=4.69)$. La edad de excarcelación a la comunidad terapéutica oscilaba entre 21 y 44 años ( $M$ $=29.28, D T=4.5)$. En relación con el nivel académico, el $12.6 \%$ no tiene ningún estudio, es decir son analfabetos 0 saben leer y escribir con muchas dificultades, el $46.2 \%$ tienen el certificado de escolaridad, el $21 \%$ ha obtenido el graduado escolar, el 5.9\% ha llegado a los niveles de Formación Profesional (I y II) y al Bachillerato Unificado Polivalente y el $3.3 \%$ tiene acceso a estudios universitarios. Con referencia al estado civil el $73.3 \%$ está soltero, el 9.2\% está casado o convive en pareja, el $15.8 \%$ está separado/divorciado y el $1.7 \%$ viudo. El periodo de seguimiento en el $45.83 \%$ de los casos fue entre 2 y 4 años después de su excarcelación, mientras que en el $54.17 \%$ fue de 5 o más años.

\section{Instrumentos y variables}

Las fuentes documentales consultadas para cada sujeto fueron tres: el Expediente Penitenciario, el Protocolo de Clasificación y Tratamiento y el Expediente Social.

En este artículo se estudiarán aquellas variables estáticas, previas al tratamiento en C. T., que han mostrado tener una relación significativa con un nuevo ingreso en prisión. Para el análisis se han considerado las siguientes variables:

Antecedentes familiares penitenciarios: hace referencia a la existencia o no de familiares próximos (padres, hermanos) con antecedentes penitenciarios. Se establecieron dos categorias: "con antecedentes penitenciarios" y "sin antecedentes penitenciarios".

Condena total formal: se registran la/s condena/s que constan en sentencia de los periodos de condena anterior (si existiera) y del periodo de condena base (condena en la que el sujeto es excarcelado en tercer grado a C. T.). Establecimos dos categorías: "condena total corta hasta 5 años" y "condena total larga superior a 5 años".

Número de ingresos en prisión previos a la condena base: se computan el número de ingresos en prisión en calidad de detenido, preventivo y penado, previos a la condena base. Establecimos dos categorías: "no ingresos previos" y "sí ingresos previos".

Edad del primer ingreso en prisión: se registró la edad del primer ingreso en prisión bien sea en calidad de detenido, preventivo o penado. Establecimos dos categorias: "edad del primer ingreso en prisión entre 16 y 21 años" y "edad del primer ingreso en prisión entre 22 y 41 años".

Regresión de grado de cumplimiento: se registra la regresión de segundo a primer grado y de tercero a segundo grado, durante la condena anterior o durante la condena base, previa a la excarcelación al centro terapéutico extrapenitenciario. Establecimos dos categorías: "consta regresión de grado" y "no consta regresión de grado".

Revocación de la libertad condicional. Se tuvieron en cuenta dos categorías, "no consta revocación de la libertad condicional" y "sí consta revocación" de la misma en algún momento de cumplimiento de condena previo a la excarcelación al centro terapéutico.

Quebrantamiento de condena. Establecimos dos categorías, "no consta quebrantamiento de condena y sí consta quebrantamiento de condena".

Forma de finalización de la condena base. Dos categorías fueron consideradas "libertad total por extinción de condena en prisión" y "libertad total en libertad condicional".

Reincidencia en la actividad delictiva: con dos categorías, "no reincide" y "sí reincide". Se asigna a cada sujeto a uno de los dos grupos según hubiera tenido algún reingreso penado en prisión por una nueva comisión de delito o falta penal a su 
excarcelación del centro penitenciario, tras la cual se realizó el seguimiento.

\section{Procedimiento}

Para realizar este estudio se solicitó permiso al Centro Directivo de Instituciones Penitenciarias. La solicitud se acompañó con una memoria del proyecto de investigación. Se nos concedió permiso por seis meses y renovable por otros seis para consultar los documentos. Los documentos fueron consultados por la autora del artículo en el Centro Penitenciario de Burgos, y se elaboró una hoja de recogida de toda la información incluida en este trabajo. Esta hoja de recogida de datos se fue construyendo, ampliando y actualizando a medida que encontrábamos en los documentos oficiales, diferentes ítems o informaciones relevantes de ser estudiadas.

\section{Análisis de datos}

Para el análisis bivariado de las variables hemos relacionado la que hemos tomado como variable de agrupación "reincidencia a su excarcelación de la condena base" con el resto de las variables. Para el análisis estadístico bivariado, se ha utilizado la prueba de Chi-cuadrado, estableciéndose el nivel crítico $p<.05$ para rechazar hipótesis nula. Los datos son presentados en tablas de contingencia. Dado que los expedientes consultados no contenían datos de algunas variables en algunos sujetos, los porcentajes se tuvieron que calcular sobre la muestra de la que se tenía la información en cada caso.

Con el objetivo de encontrar los factores que mejor explican la reincidencia, hemos realizado un análisis de regresión logística, incluyendo como predictores aquellas variables que mostraron una relación significativa con la reincidencia a nivel bivariado. Para realizar todos estos análisis estadísticos se ha utilizado el paquete estadístico PASW 18 - SPSS.

\section{Resultados}

Todas las variables analizadas en este estudio mostraron una relación estadísticamente significativa con la reincidencia. En primer lugar, observamos que un menor porcentaje de internos reincidia en la actividad delictiva cuando éstos no tenían antecedentes familiares penitenciarios en comparación con los internos que sí los tenían. (Tabla 1).

Tabla 1. Relación Antecedentes Familiares Penitenciarios y Reincidencia

\begin{tabular}{ccccc}
\hline \multirow{2}{*}{ Antecedentes familiares penitenciarios } & \multicolumn{3}{c}{ Reincidencia } \\
\cline { 3 - 5 } & Frecuencia & No & Si & Total \\
\hline \multirow{2}{*}{ No } & $\%$ & $75,9 \%$ & $24,1 \%$ & 87 \\
& Frecuencia & 14 & 12 & 26 \\
Si & $\%$ & $53,8 \%$ & $46,2 \%$ & $100,0 \%$ \\
& Frecuencia & 80 & 33 & 113 \\
Total & $\%$ & $70,8 \%$ & $29,2 \%$ & $100,0 \%$ \\
& & & &
\end{tabular}

$X^{2}(1)=4.693, p=.030$
La condena total formal también mostró una relación significativa con la reincidencia. Un mayor porcentaje de internos con condenas superiores a cinco años reincidia en la actividad delictiva. (Tabla 2).

Tabla 2. Relación Condena Total Formal y Reincidencia

\begin{tabular}{ccccc}
\hline \multirow{2}{*}{ Condena total } & & \multicolumn{3}{c}{ Reincidencia } \\
\cline { 3 - 5 } & & No & Si & Total \\
\hline \multirow{2}{*}{ Condena total hasta 5 años } & Frecuencia & 32 & 7 & 39 \\
& $\%$ & $82,1 \%$ & $17,9 \%$ & $100,0 \%$ \\
Condena total superior 5 años & Frecuencia & 47 & 27 & 74 \\
& $\%$ & $63,5 \%$ & $36,5 \%$ & $100,0 \%$ \\
Total & Frecuencia & 79 & 34 & 113 \\
& $\%$ & $69,9 \%$ & $30,1 \%$ & $100,0 \%$ \\
\hline
\end{tabular}

$X^{2}(1)=4.172, p=.041$

Asimismo, encontramos que los internos que reinciden tienen un mayor número de ingresos previos en comparación con los que no reinciden. (Tabla 3).

Tabla 3. Relación Ingresos en Prisión Previos a la Condena Base y Reincidencia

\begin{tabular}{ccccc}
\hline \multirow{2}{*}{ Ingresos previos en prisión } & \multicolumn{3}{c}{ Reincidencia } \\
\cline { 3 - 5 } & & No & Si & Total \\
\hline \multirow{2}{*}{ Sin ingresos previos } & Frecuencia & 59 & 17 & 76 \\
& $\%$ & $77,6 \%$ & $22,4 \%$ & $100,0 \%$ \\
Con ingresos previos & Frecuencia & 22 & 17 & 39 \\
& $\%$ & $56,4 \%$ & $43,6 \%$ & $100,0 \%$ \\
Total & Frecuencia & 81 & 34 & 115 \\
& $\%$ & $70,4 \%$ & $29,6 \%$ & $100,0 \%$ \\
\hline
\end{tabular}

$X^{2}(1)=5.574, p=.018$

Respecto a la edad del primer ingreso en prisión y la reincidencia, es de destacar que los internos que tuvieron su primer ingreso en prisión entre 16 y 21 años reincidieron en la actividad delictiva en mayor medida que los que ingresaron con 22 años en adelante. (Tabla 4).

Tabla 4. Relación Edad del Primer Ingreso en Prisión y Reincidencia

\begin{tabular}{ccccc}
\hline \multirow{2}{*}{ Edad del primer ingreso en prisión } & \multicolumn{3}{c}{ Reincidencia } \\
\cline { 3 - 5 } & & No & $\mathrm{Si}$ & Total \\
\hline \multirow{2}{*}{ Entre 16 y 21 años } & Frecuencia & 36 & 24 & 60 \\
& $\%$ & $60,0 \%$ & $40,0 \%$ & $100,0 \%$ \\
\hline \multirow{2}{*}{ Entre 22 y 41 años } & Frecuencia & 46 & 10 & 56 \\
& $\%$ & $82,1 \%$ & $17,9 \%$ & $100,0 \%$ \\
\hline \multirow{2}{*}{ Total } & Frecuencia & 82 & 34 & 116 \\
& $\%$ & $70,7 \%$ & $29,3 \%$ & $100,0 \%$ \\
\hline
\end{tabular}

$X^{2}(1)=6.854, p=.009$

Por otro lado, también observamos una relación significativa entre regresión en el grado de cumplimiento y reincidencia, en el siguiente sentido: los internos que tuvieron que 
ser regresados en el grado de cumplimiento reinciden más que los que no fueron regresados. (Tabla 5).

Tabla 5. Relación regresión de grado de cumplimiento y reincidencia

\begin{tabular}{ccccc}
\hline \multirow{2}{*}{$\begin{array}{c}\text { Regresión de grado de cumplim- } \\
\text { iento }\end{array}$} & \multicolumn{3}{c}{ Reincidencia } \\
\cline { 3 - 5 } No & Frecuencia & 56 & Si & Total \\
\hline \multirow{2}{*}{ Si } & $\%$ & $76,7 \%$ & $23,3 \%$ & 73 \\
& Frecuencia & 22 & 16 & 38 \\
\hline \multirow{2}{*}{ Total } & $\%$ & $57,9 \%$ & $42,1 \%$ & $100,0 \%$ \\
& Frecuencia & 78 & 33 & 111 \\
& $\%$ & $70,3 \%$ & $29,7 \%$ & $100,0 \%$ \\
\hline
\end{tabular}

$X^{2}(1)=4.236, p=.040$

Del mismo modo, un mayor porcentaje de internos reincidía en la actividad delictiva cuando éstos habían protagonizado revocación de la libertad condicional en comparación con los internos que no habían protagonizado revocación de la libertad condicional. (Tabla 6).

Tabla 6. Relación Revocación de la Libertad Condicional y Reincidencia

\begin{tabular}{ccccc}
\hline \multirow{2}{*}{$\begin{array}{c}\text { Revocación de la libertad } \\
\text { condicional }\end{array}$} & No & Si & Total \\
\cline { 3 - 5 } No & Frecuencia & 52 & 15 & 67 \\
& $\%$ & $77,6 \%$ & $22,4 \%$ & $100,0 \%$ \\
\hline \multirow{2}{*}{ Si } & Frecuencia & 8 & 8 & 16 \\
& $\%$ & $50,0 \%$ & $50,0 \%$ & $100,0 \%$ \\
\hline \multirow{2}{*}{ Total } & Frecuencia & 60 & 23 & 83 \\
& $\%$ & $72,3 \%$ & $27,7 \%$ & $100,0 \%$ \\
\hline
\end{tabular}

$X^{2}(1)=4.196, p=.027$

En cuanto a la relación entre el quebrantamiento de condena (e.g., aprovechar un permiso penitenciario para fugarse o cometer un delito) y la reincidencia, observamos que los porcentajes más altos de reincidencia se encontraban en el grupo que habían cometido delito de quebrantamiento en comparación con los que no lo habian cometido. (Tabla 7).

Tabla 7. Relación Quebrantamiento de Condena y Reincidencia

\begin{tabular}{ccccc}
\hline \multirow{2}{*}{ Quebrantamiento de condena } & \multicolumn{3}{c}{ Reincidencia } \\
\cline { 3 - 5 } & Frecuencia & No & Si & Total \\
\hline \multirow{2}{*}{ No } & $\%$ & $75,6 \%$ & $24,4 \%$ & $100,0 \%$ \\
\hline \multirow{2}{*}{ Si } & Frecuencia & 13 & 12 & 25 \\
& $\%$ & $52,0 \%$ & $48,0 \%$ & $100,0 \%$ \\
\hline \multirow{2}{*}{ Total } & Frecuencia & 78 & 33 & 111 \\
& $\%$ & $70,3 \%$ & $29,7 \%$ & $100,0 \%$ \\
\hline
\end{tabular}

$X^{2}(1)=5.156, p=.023$

También encontramos una relación estadísticamente significativa entre la forma de finalizar la condena base y la reincidencia. Los internos que finalizan la condena base en libertad condicional reinciden menos que los internos que finalizan la condena base en libertad total por extinción de condena. (Tabla 8).

Tabla 8. Relación Forma de Finalización de la Condena Base y Reincidencia

\begin{tabular}{ccccc}
\hline \multirow{2}{*}{ Forma de finalización de la condena base } & \multicolumn{3}{c}{ Reincidencia } \\
\cline { 3 - 5 } & & No & Si & Total \\
\hline \multirow{2}{*}{ Extinción de condena } & Frecuencia & 7 & 12 & 19 \\
& $\%$ & $36,8 \%$ & $63,2 \%$ & $100,0 \%$ \\
\hline \multirow{2}{*}{ Libertad Condicional } & Frecuencia & 45 & 12 & 57 \\
& $\%$ & $78,9 \%$ & $21,1 \%$ & $100,0 \%$ \\
\hline \multirow{2}{*}{ Total } & Frecuencia & 52 & 24 & 76 \\
& $\%$ & $68,4 \%$ & $31,6 \%$ & $100,0 \%$ \\
\hline
\end{tabular}

$X^{2}(1)=11.692, p=.001$

Finalmente, realizamos un análisis de regresión logística siguiendo el método Wald hacia adelante (paso a paso) para ver qué variables explicaban en mayor medida el fenómeno de la reincidencia. En este análisis se incluyeron todas las variables que habian mostrado una relación significativa con la reincidencia en las tablas de contingencia, exceptuando la revocación de la libertad condicional y la forma de finalización de la condena puesto que no todos los participantes podian verse afectados por ellas (algunos internos no estaban en situación de poder beneficiarse de la obtención de la libertad condicional y otros no habian finalizado su condena aún como para estudiar la forma de finalización).

La única variable que resultó significativa en el análisis fue la edad del primer ingreso en prisión (Wald $=6,692$, $p$ $=, 010)$, explicando un $9,4 \%$ de la variable criterio $\left(R^{2}\right.$ de Nagelkerke $=$,094). En este sentido, la probabilidad de reincidencia era 3,274 (OR) veces mayor en el grupo con edad de ingreso más joven (16-21 años) con respecto al más mayor (22-41 años).

\section{Discusión}

De acuerdo con nuestro objetivo, hemos encontrado relaciones significativas entre reincidencia y las siguientes variables: antecedentes familiares penitenciarios, condena total formal, número de ingresos en prisión, regresión en el grado de cumplimiento, revocación de la libertad condicional, quebrantamiento de condena, finalización de la condena base en prisión y especialmente con edad del primer ingreso.

En cuanto a la relación entre reincidencia y antecedentes familiares penitenciarios, los datos reflejan que cuando un padre, madre o hermanos manifiestan conducta delictiva hay más probabilidades de que otros miembros de la familia imiten dicha conducta. Esto corrobora lo obtenido en otros estudios (Becedóniz et al., 2009; Serrano et al., 2001). Sin embargo pensamos que la valoración de si una persona se puede beneficiar o no de finalizar su condena en una C. T. no se puede basar en aspectos como que algún familiar suyo haya cometido un delito. No obstante consideramos que un 
objetivo del tratamiento en C. T. tiene que ser que el sujeto tome conciencia de cómo las relaciones antisociales (incluyendo a padres, hermanos, amigos y otros significativos) es un factor de riesgo tanto para involucrarse en la carrera delictiva como en la recaída en el consumo de sustancias. Las relaciones antisociales o prosociales junto con las actitudes, valores y creencias prodelictivas 0 antidelictivas son factores de riesgo y protección respectivamente más relevantes que la persona tiene disponible en cualquier situación. Son estas variables las que hay que tener en cuenta, entre otras, a la hora de dar un juicio sobre el riesgo de reingresar en prisión y las que se reconocen en modelos explicativos como el modelo integrado de la conducta de Andrews y Bonta (2003).

En relación a la edad del primer ingreso en prisión, el número de ingresos previos y la condena total formal, encontramos un mayor porcentaje de personas que reincidian cuanto más jóvenes eran en su primer ingreso, si presentaban un mayor número de ingresos previos a la condena base, y si se daba una condena total formal más larga. Estos resultados son consistentes con estudios previos (Luque et al., 2004a, 2004b; Paíno et al., 1996; Redondo et al., 1994; Roca y Caixal, 2002, 2005; Serrano et al., 2001).

Estas variables podrian estar asociadas de manera conjunta con la reincidencia y el posterior reingreso en prisión, explicando un posible proceso: los internos que entran más jóvenes en prisión por primera vez suelen cumplir condenas cortas, saliendo de prisión con un alto potencial delictivo y reingresando nuevamente en diferentes ocasiones. A medida que pasa el tiempo, al ser reincidente, las condenas son cada vez más largas, aumentándose el tiempo de condena total formal. En el análisis conjunto de los predictores de la reincidencia y posterior reingreso en prisión, la edad de primer ingreso en prisión es el único factor que predice de manera significativa el volver a prisión una vez excarcelado a C. T. En este sentido, el hecho de pertenecer al grupo de edad más joven en el primer ingreso en prisión (16 a 21 años) hace que la probabilidad de reincidencia sea tres veces mayor en comparación con pertenecer al grupo con edad de entrada mayor (22 a 41 años). Estos resultados pueden estar relacionados, sería necesario analizarlo cuidadosamente, con otra variable que es la edad de excarcelación al centro de tratamiento. Encontramos estudios en los cuales los participantes de mayor edad reingresaron en prisión más tarde o no reingresaron en comparación con el grupo de menor edad (Inciardi et al., 2004; Prendergast, Hall, Wexler, Melnick y Cao, 2004). Este resultado es apoyado por la literatura científica que propone un proceso de "maduración" en los delincuentes habituales toxicómanos, según el cual el control individual de la adicción está relacionado con el logro de un nivel adecuado de maduración y estabilidad personal (Asociación Americana de Psiquiatría, 2002). Es decir, la conducta desviada, incluyendo el delito y el uso de drogas, decrecerá según el adicto se vuelve más viejo.

A continuación queremos analizar cómo se relacionan con la variable reingreso en prisión, después del tratamiento en C. T. por comisión de delito, algunas consecuencias en el régimen de vida del recluso. Algunas de estas consecuencias por mala conducta del interno, entre otras, pueden ser la regresión en el grado de cumplimiento, la revocación de la libertad condicional y el quebrantamiento de condena.

En la práctica determinados comportamientos como no reingreso tras el disfrute de permisos de salida, o volver tarde o en malas condiciones (bajo los efectos de las distintas drogas), hacer un mal uso del permiso (comisión de un presunto delito), incumplimiento de las condiciones impuestas por el régimen de vida (horarios de salida, irregularidades manifiestas en la actividad laboral o en el programa de tratamiento de drogodependencias $u$ otros), tener partes sancionadores sin cancelar, intento de evasión, etc., pueden ser los responsables de las consecuencias enumeradas anteriormente. Consecuencias todas ellas que afectarían en mayor o menor medida lo que autores como Redondo et al. (1994), denominan penosidad $u$ opresividad del tiempo de encarcelamiento de un sujeto.

Según los resultados de nuestra investigación la regresión en el grado de cumplimiento (regresión de régimen de vida ordinario a régimen cerrado, o regresión de régimen de vida abierto a régimen de vida ordinario), la revocación de la libertad condicional y el quebrantamiento de condena se relacionan de manera significativa con la variable reingreso en prisión tras el tratamiento en $\mathrm{C}$. T. al que fueron sometidos los sujetos excarcelados.

Nuestros resultados coinciden con los obtenidos en otras investigaciones como la de Redondo et al., (1994) y Roca y Caixal, $(2002,2005)$ que concluyen que las personas que reincidieron a diferencia de las que no reincidieron se beneficiaron en menor medida de reducciones de condena por el trabajo y sufrieron regímenes de vida más duros o penosos. En la misma línea Serrano et al., (2001) concluyen que todas las medidas que influyen en unas condiciones más suaves de cumplimiento como: reducciones de condena, clasificación en tercer grado de tratamiento, permisos de salida etc., resultan estar relacionadas con una menor reincidencia, lo que resulta esperanzador, ya que nuestra legislación contempla esas actuaciones y pueden ser el instrumento adecuado para reducir la reincidencia en el delito.

Los resultados obtenidos en nuestro trabajo señalan que el hecho de acceder a la libertad condicional se asocia con menor reincidencia. El hecho de extinguir la condena en prisión es indicativo de mala adaptación al internamiento y tratamiento penitenciario. La experiencia a nivel laboral en este ámbito de una de las autoras nos facilita el plantear que esta situación no siempre se explica por una nueva comisión de delito sino por incumplimientos durante el tratamiento en C. T., Estos incumplimientos se traducen en expulsiones, abandonos y/o la decisión voluntaria de volver a prisión. En realidad algunos de los sujetos enviados a $C$. T. por el sistema penitenciario prefieren la prisión al tratamiento en $\mathrm{C}$. T. El contexto normativo y la intensidad del tratamiento colocan a la persona en una situación de estrés que no siempre es bien gestionada. En este sentido pensamos que es importante poder determinar la idoneidad de la tipología del recurso o tratamiento (ambulatorio, centro de día, C. T.) en función de las características del sujeto. Esto facilitaría en la medida de lo posible, que el paciente tuviese una mejor adaptación 
al tratamiento que le permita finalizar el programa, dado que esta variable resulta relevante respecto a la reincidencia y posterior reingreso en prisión.

En definitiva, constatamos que el haber accedido a la libertad condicional se relaciona con una menor reincidencia futura. Ahora bien, nada permite conocer si es que sólo se aplica la libertad condicional a aquellos sobre los que se intuye buen pronóstico, o si por el contrario, el propio hecho de que se confie en algunos sujetos proporcionándoles el beneficio de disfrutar de la libertad condicional, hace que se reduzca el riesgo de su reincidencia.

Nuestros resultados son confirmados por Luque et al., (2004a, 2004b, 2005) que afirman que entre los reincidentes es mayor el colectivo que fue excarcelado de la condena base en libertad definitiva que en libertad condicional.

Como toda investigación, este estudio no está exento de limitaciones. Una de ellas se relaciona con la pérdida de puntuaciones de algunos sujetos en algunas variables debido a la carencia de esta información en las fuentes documentales consultadas. Este aspecto puede debilitar el análisis conjunto de las variables que se asocian con la reincidencia. Sin embargo, este último aspecto no parece resultar tan problemático al observar que los resultados encontrados confirman estudios anteriores realizados con estas mismas variables.

Por otro lado el periodo de seguimiento en 55 sujetos es inferior a cinco años, aunque nosotros hemos intentado tomar como referencia un periodo de seguimiento de cinco años al igual que otros estudios realizados en España (Roca y Caixal, 2002, 2005) y en Estado Unidos (Prendergast, Hall, Wexler, Melnick y Cao, 2004; Wormith et al., 2007; Zhang, Roberts y McCollister, 2011). Sin embargo, la mayor parte de la muestra se sitúa por encima del periodo de seguimiento de 5 años. Queda pendiente en posteriores investigaciones establecer un criterio único de seguimiento, recogiendo la variable reincidencia de estos 55 sujetos una vez que han transcurridos 5 años desde su excarcelación.

Otra posible limitación es la generalización de los resultados. Los internos provenían de distintas prisiones de Castilla León, prisiones de las cuales eran excarcelados a la C. T. correspondiente, donde la Junta de Castilla y León les había concedido una plaza subvencionada. El Centro Penitenciario de Burgos custodia los documentos oficiales consultados, por ser el centro de referencia a nivel penitenciario responsable del seguimiento de la medida alternativa a la pena privativa de libertad, por lo que pensamos que estos datos podrían extrapolarse a dicha comunidad.

Otra limitación es que este trabajo aborda tan solo el estudio de las variables previas al programa en C. T. y no modificables por el tratamiento. Bajo nuestro punto de vista, variables como la edad de primer ingreso en prisión, tener antecedentes familiares penitenciarios, protagonizar estancias largas y numerosos ingresos en prisión entre otros factores analizados en este estudio, son datos que tienen mucho peso en el expediente del recluso y que no se pueden borrar. En Ramos y Carcedo (2010) se describe la relación entre variables de resultado del tratamiento (tiempo de permanencia en el programa de C. T., finalización del tratamiento con/sin alta terapéutica), variables previas al tratamiento (los hábitos laborales, características de personalidad como la impulsividad, afrontamiento de la patología infecciosa por VIH) y la variable dependiente reingreso en prisión, remitimos al lector interesado la consulta a este documento. Este último conjunto de variables independientes creemos que son susceptibles de ser modificadas por el tratamiento. Sin embargo queda por estudiar conjuntamente qué variables son más relevantes para predecir la reincidencia de reclusos drogodependientes excarcelados en tercer grado a tratamiento extrapenitenciario. Creemos especialmente necesario, en la misma línea que han apuntado Holloway, Bennett y Farrington (2006) y Wormith et al. (2007) estudiar la relación existente entre la reincidencia y otras variables del proceso del tratamiento, como son la preparación y la motivación para el cambio, la gravedad del trastorno por abuso de sustancias, la adherencia al tratamiento, la relación terapéutica, y los apoyos externos, con el objetivo de mejorar la intervención y prevenir con mayor éxito la reincidencia en la actividad delictiva.

En nuestra investigación hemos medido la reincidencia penitenciaria que según Capdevila y Ferrer (2009) es entendida como el reingreso de una persona que ha sido excarcelada por el cumplimiento de una pena privativa de libertad, a cumplir nuevamente una medida de privación de libertad en la cárcel. En esta tasa de reincidencia perdemos todos aquellos casos que han cometido delito pero no han sido detenidos y aquellos casos que habiendo sido detenidos y procesados por un nuevo hecho delictivo no han sido condenados a pena privativa de libertad en prisión. Nuestro sistema penal recoge diferentes tipos de pena que no implican privación de libertad en una prisión (Segovia, 2004). Sería necesario, en futuras investigaciones, medir la reincidencia de una manera más amplia a través de autoinforme de actividad delictiva y consultando los antecedentes penales que existen en las bases de datos de gestión estatal, al igual que hacen otros estudios (Sacks, Chaple, Sacks, McKendrick y Cleland, 2012; Sacks, McKendrick, Sacks, Banks y Harle, 2008; Sacks, Sacks, McKendrick, Banks y Stommel, 2004; Sullivan, McKendrick, Sacks y Banks, 2007).

Otras futuras líneas de investigación son: comparar la tasa de encarcelamiento obtenida en nuestra investigación (sujetos excarcelados en tercer grado de cumplimiento de condena a tratamiento en C. T. extrapenitenciaria) con la tasa de un nuevo reingreso en prisión obtenido con otras modalidades de tratamiento comunitario de menor intensidad en la intervención y por tanto de más bajo coste (tratamiento ambulatorio, tratamiento en centro de dia), o con el reingreso en prisión de aquellos drogodependientes que no han seguido ningún tratamiento (grupo de no intervención o no tratamiento). Con el objeto de evaluar la eficacia de diferentes programas. Al respecto queremos hacer hincapié que las modalidades de tratamiento que existen en la comunidad, fuera del entorno carcelario, existen también dentro de algunas prisiones. En este sentido queremos destacar que una de las modalidades de tratamiento que a partir de los años 90 se ha desarrollado en las prisiones españolas son las C. T. intrapenitenciarias. Aquellos sujetos que, habiendo solicitado su 
excarcelación a C. T. en tercer grado de cumplimiento, no se les concedió la medida alternativa entre otras razones por presentar un perfil de alto riego de no hacer un buen uso de la excarcelación, tienen la opción de integrarse en tratamientos intrapenitenciarios. En este punto queremos señalar que la investigación ha demostrado que sin un tratamiento continuado (tratamiento en prisión seguido de tratamiento en la comunidad) se puede esperar que un gran porcentaje de los delincuentes drogodependientes vuelvan a caer en pautas de consumo crónico de drogas y de delincuencia a los pocos meses de haber salido de prisión (Wexler y Prendergast, 2010).

Estudios realizados fundamentalmente en EEUU, pionera en este campo de investigación (Inciardi et al., 2004; Prendergast, Hall, Wexler, Melnick y Cao, 2004) concluyen que los programas de tratamiento de la drogodependencia con reclusos en régimen de semilibertad (o tercer grado penitenciario) y en libertad condicional facilitan la transición de la cárcel a la comunidad, de tal manera que factores como la edad de excarcelación y tener un tratamiento continuado son los factores que más predicen menos recaídas en el consumo y menor número de reingreso en prisión.

Este artículo supone una aportación, dada la escasez de investigación, al estudio de la reincidencia y reingreso en prisión con población penitenciaria drogodependiente que se beneficia de una excarcelación en tercer grado para seguir cumpliendo condena en un centro de tratamiento. La recuperación de la adicción a las drogas es un proceso continuo. Aunque la participación en el tratamiento puede comenzar en el entorno carcelario, es de vital importancia la atención posterior y continuada. Por lo tanto, un modelo de rehabilitación de delincuentes drogodependientes deberá incluir el tratamiento dentro de prisión, el tratamiento durante los periodos de transición (excarcelación en tercer grado y libertad condicional) y el tratamiento posterior o continuado una vez que la persona está en libertad total.

\section{Reconocimientos}

Queremos agradecer al Centro Penitenciario de Burgos y al Servicio Social Penitenciario Externo por su ayuda tanto en el acceso y la recogida de la información como por su disponibilidad en todo momento. Los autores desean expresar su agradecimiento a las aportaciones en asesoramiento metodológico a los profesores Silvia Ubillos Landa y José Luis González Castro.

\section{Conflicto de intereses}

Los autores expresan que no existe ningún conflicto de intereses que declarar.

\section{Referencias}

Andrews, D. A. y Bonta, J. (2003). The psychology of criminal conduct (3rd ed.). Cincinnati: Anderson Publishing Co.

Asociación Americana de Psiquiatría (2002). DSM-IV-TR. Manual diagnóstico y estadístico de los trastornos mentales. Texto revisado. Barcelona: Masson

Bahr, S. J., Masters, A. L. y Taylor, B. M. (2012). What works in substance abuse treatment programs for offenders? Prison Journal, 92, 155174. doi:10.1177/0032885512438836.

Becedóniz, C., Rodríguez, F. J., Herrero, F. J., Paíno, S. G. y Chan, E. C. (2005). Reincidencia de menores infractores en la carrera delictiva. Investigando factores de la problemática familiar. En J. Sobral, G. Serrano y J. Regueiro (Comp.), Psicología jurídica de la violencia y de género (pp.99-108). Madrid: Biblioteca Nueva.

Bravo, A., Sierra, M. J. y Del Valle, J. F. (2009). Evaluación de resultados de la ley de responsabilidad penal de menores. Reincidencia y factores asociados. Psicothema, 21, 615-621.

Burdon, W. M., Messina, N. P. y Prendergast M. L. (2004). The California treatment expansion initiative: aftercare participation, recidivism, and predictors of outcomes. The Prison Journal, 8, 61-80. doi:10.1177/0032885503262455.

Capdevila, M y Ferrer, M. (2009). Taxa de reincidència penitenciària 2008. Recuperado el 17 de septiembre de 2009, desde http:// www20.gencat.cat/docs/Justicia/Documents/ ARXIUS/SC-1-07609_cat.pdf.

Cid, J. (2007). ¿Es la prisión criminógena? Un análisis comparativo de reincidencia entre la pena de prisión y la suspensión de la pena. Revista de Derecho Penal y Criminología, 19, 427-456.

Elzo, J., Huete, J., Laespada, M. T. y Santibáñez, R. (1995). Alternativas terapéuticas a la prisión en delincuentes toxicómanos. Un análisis de historias de vida. Vitoria- Gasteiz: Servicio Central de Publicaciones del Gobierno Vasco.

Fernández, C. (2005). Una evaluación de necesidades, procesos y satisfacción de drogodependientes con y sin problemática judicial tratados en comunidades terapéuticas. Adicciones, 17, 301-305.

Fernández, J. y Rico, A. (2004). Evaluación del tratamiento de rehabilitación Proyecto Hombre, para drogodependientes en situación de cumplimiento extrapenitenciario. En J. C. Menéndez y A. Rico (autores), Drogodependencias y justicia. Evaluación del tratamiento de drogodependientes con causas penales en Proyecto Hombre de Asturias (pp. 127-129). Oviedo: Gráficas Oviedo.

Fiestas, F. y Ponce, J. (2012). Eficacia de las comunidades terapéuticas en el tratamiento de problemas por uso de sustancias psicoactivas: una revisión sistemática. Revista Peruana de Medicina Experimental y Salud Pública, 29, 12-20. 
Gañán, A. y Gordon, B. (1999). Las drogodependencias en los sistemas judicial y de tratamiento. Divergencias y puntos de encuentro. Trastornos adictivos, 1, 205-210.

Garrido, V., Stangeland, P. y Redondo, S. (2001). Principios de Criminología. Valencia: Tirant Lo Blanch.

Gendreau, P., Little, T. y Goggin, C. (1996). A meta-analysis of the predictors of adult offender recidivism: what works? Criminology, 34, 575-607.

González, I. (2009). Abolicionismo, cárceles e inseguridad ciudadana. Crítica, alternativas y tendencias. Revista de Derecho Penal y Criminología, 1, 291-315.

Holloway, K. R., Bennett, T. H. y Farrington, D. P. (2006). The effectiveness of drug treatment programs in reducing criminal behaviour: A meta-analysis. Psicothema, 18, 620-629.

Inciardi, J. A., Martín, S. S. y Butzin C. A. (2004). Five-year outcomes of therapeutic community treatment of drug-involved offenders after release from prison. Crime \& Delinquency, 50, 88-107. doi:10.1177/0011128703258874.

Leganés, S. (2005). La evolución de la clasificación penitenciaria. Madrid: Ministerio de Interior.

López, V. M. (2006). La responsabilidad civil en la L. 0. 7/2003 y su incidencia en el tratamiento penitenciario. Revista de estudios penitenciarios, 252, 77-116.

Luque, E., Ferrer, M. y Capdevila, M. (2004a). La reincidència penitenciària a Catalunya (1997- 2002). Justidata 37. Barcelona: Centro d'Estudis Jurídics i Formació Especialitzada.

Luque, E., Ferrer, M. y Capdevila, M. (2004b). La reincidencia. Invesbreu 26. Barcelona: Centro d'Estudis Jurídics i Formació Especialitzada.

Luque, E., Ferrer, M. y Capdevila, M. (2005). La reincidencia penitenciaria en Cataluña. Justícia i Societat, 25, 15-16.

Menéndez, J. C. y Rico, A. (2004). Drogodependencias y justicia. Evaluación del tratamiento de drogodependientes con causas penales en Proyecto Hombre de Asturias. Oviedo: Gráficas Oviedo.

Ministerio del Interior (2004). Normativa penitenciaria. Revista de estudios penitenciarios, 250, 192-197.

Paino, S. G., Rodríguez, F. J., y Cuevas, L. M. (1996). Indicadores de riesgo en la reincidencia. Cuadernos de política criminal, 60, 77187.

Pérez, E. y Redondo, S. (1991). Efectos psicológicos de la estancia en prisión. Papeles del Psicólogo, 48, 54-57.

Prendergast, M. L., Hall, E. A., Wexler, H. K., Melnick, H. y Cao, Y. (2004). Amity prison-based therapeutic community: 5-year outcomes. The Prison Journal, 84, 36-60. doi:10.1177/0032885503262454.

Ramos, V. y Carcedo, R. (2010). Factores dinámicos de la reincidencia de reclusos drogodependientes en tratamiento en comunidad terapéutica. Behavioral Psychology, 18, 613-627.

Real Decreto 190/1996, de 9 de febrero, por el que se aprueba el Reglamento Penitenciario de 1996. En B.0.E. núm. 40 de 15 de febrero de 1996 (pp.5380-5435).
Redondo, S., Funes, J. y Luque, E. (1994). Justicia penal y reincidencia. Barcelona: Centre d'Estudis Jurídics i Formació Especialitzada de la Generalitat de Catalunya y Fundació Jaume Callis.

Roca, X. y Caixal G. (2002). Estudi de la reincidència en una mostra d'interns penitenciaris sotmesos a tractament per l'addicció a drogues. Invesbreu, 21, 4-8.

Roca, X. y Caixal G. (2005). Análisis de los factores estáticos de reincidencia en el tratamiento de comunidad terapéutica. Invesbreu, 28, 8-12.

Serrano, J., Romero, A. y Noguera, M. L. (2001). Variables criminológicas y reincidencia. En Ministerio del Interior (Ed.) Estudios e investigaciones de la Central Penitenciaria de Observación (pp. 173-276). Madrid: Ministerio del Interior.

Sacks, S., Chaple, M., Sacks, J. Y., McKendrick, K. y Cleland, C. M. (2012). Randomized trial of a reentry modified therapeutic community for offenders with co-occurring disorders: Crime outcomes. Journal of Substance Abuse Treatment, 42, 247-259. doi:10.1016/j.jsat.2011.07.011.

Sacks, S., McKendrick, K., Sacks, J. Y., Banks, S. y Harle, M. (2008). Enhanced outpatient treatment for co-occurring disorders: Main outcomes. Journal of Substance Abuse Treatment, 34, 48-60. doi:10.1016/j.jsat.2007.01.009.

Sacks, S., Sacks J. Y., McKendrick, K., Banks, S. y Stommel, J. (2004). Modified TC for MICA offenders: Crime outcomes. Behavioral Sciences \& The Law, 22, 477-501. doi:10.1002/bsl.599.

Segovia, J. L. (2004). Código Penal y todas sus reformas al alcance de todos. Madrid: Editorial Popular.

Sullivan, C. J., Mckendrick, K., Sacks, S. y Banks, S. (2007). Modified therapeutic community treatment for offenders with MICA disorders: Substance use outcomes. American Journal of Drug and Alcohol Abuse, 33, 823-832. doi: 10.1080/00952990701653800.

Tournier, P. (2007). Hacia una aproximación global de la cuestión de las alternativas a la detención. Revista de Derecho Penal y Criminología, 19, 523-530.

Wexler, H. K., De Leon, G., Thomas, G., Kressel, D. y Peters J. (1999). The amity prison TC evaluation. Reincarceration Outcomes. Criminal Justice and behaviour, 26, 147-167.

Wexler, H. K. y Prendergast, M. L. (2010). Therapeutic communities in United States prisons: Effectiveness and challenges. Therapeutic Communities: the International Journal for Therapeutic and Supportive Organizations, 31, 157-175.

Wexler, H. K., Falkin, G. P. y Lipton, D. S. (1990). Outcome evaluation of a prison therapeutic community for substance abuse treatment. Criminal Justice and Behavior, 17, 71-92.

Wormith, J. S., Althouse, R., Simpson, M., Reitzel, L. R., Fagan, T. J. y Morgan, R. D. (2007). The rehabilitation and reintegration of offenders. The Current Landscape and Some Future Directions for Correctional Psychology. Criminal Justice and Behavior, 34, 879892. doi:10.1177/0093854807301552.

Zhang, S. X., Roberts, R. E. L. y McCollister, K. E. (2011). Therapeutic Community in a California Prison. Treatment Outcomes After 5 Years. Crime \& Delinquency, 57, 82-101. doi: $10.1177 / 0011128708327035$. 
